\title{
City-Size Distribution and Industry Agglomeration: An Empirical Analysis Based on the County - level in Beijing-Tianjin-Hebei Region
}

\author{
Heng Tian \\ Tianjin University of Finance and Economics, Tianjin, 300222, China
}

Keywords: City-Size Distribution, Industry Agglomeration, Zipf Law

\begin{abstract}
The urban agglomeration of the Beijing-Tianjin-Hebei region is the core development engine of the Bohai Economic Circle area. However, over the years, because of regional and local competition, the level of integration is low in the city circle, the gap is large in the region, the flow and distribution of population is extremely uneven, which is described as "the coexistence of advanced European cities and backward rural Africa" by educational circles. In the 2014, the cooperative development among Beijing-Tianjin-Hebei has become a major national strategy, highlighting the urgent realistic needs of "To build a new capital economic circle for facing the future and promote the innovation of regional development system". The country has established national new district in Xiongan in 2017, which is a millennium plan and national affairs. Besides, as the key content in research of the coordinated development among the three cities, the research on the optimization of population distribution and the realization of system is not only has the urgent need of reality, but also has important reference value to explore the theory and practice mode of China's regional integration in the future.
\end{abstract}

\section{Introduction}

The coordinated development for the Beijing-Tianjin-Hebei region,in 2014, proves to be the major national strategy.With administrative division and local competition, this region has had low integration level, big development gap, large energy consumption and environmental cost for years. The motivation and distribution of population has been unbalanced as well. On account of the great population expansion, the "big city malaise", such as traffic jam, environmental pollution and the overload of schools and hospitals has become more and more serious. On the other hand, the economy weak and backward production capacity result in low economies of scale and population absorbing capacity,in Hebei province.On April 1, 2017, China set Xiongan as national new district, which is regarded as a millennium plan. The establishment of Xiongan is of great importance for dispersing intensively the non-capital functions of Beijing, exploring the new mode of development in densely inhabited district, adjusting the urban layout and spatial structure of this region and developing an innovation-driven growth engine. This is the key of the 13th Five Year Plan in November, 2015, which includes developing several growth engines driving regional coordinated development, optimizing urban spatial layout and industrial structure in order to prevent "the metropolitan malaise". The establishment of Xiongan is also in the context of the policy that continue advancing new type of human-centered urbanization put forward in the Central Economic Working Conference in December, 2016.

\section{The characteristics of population distribution in Beijing - Tianjin - Hebei region}

The resident population has growth constantly, and the population flowing into Beijing and Tianjin has increased, with each passing year.

The Beijing-Tianjin-Hebei region includes 2 municipalities, 11 municipal districts, 20 county-level cities and 117 counties. It is estimated that 1.195 million floating population, on 
average, entries into the region since 2007, the population size of this area has reached 110 million people in 2014. Since 2011, the growth rate of resident population in Beijing-Tianjin-Hebei region has declined, the total size of the resident population in this region, however, is still increasing every year. Figure 1 shows the total size and annual growth rate of the resident population of the Beijing-Tianjin-Hebei region from 2007 to 2014.

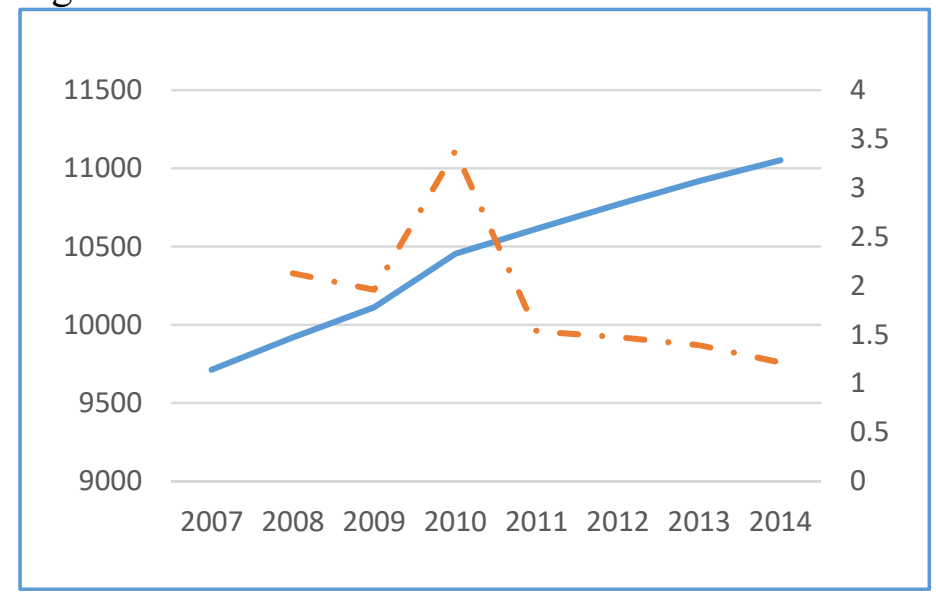

Figure 1: the total population of Beijing-Tianjin-Hebei (million) and resident population growth rate $(\%)$

Table1: The proportion of population in the Beijing-Tianjin-Hebei region(\%)

\begin{tabular}{c|c|c|c}
\hline & Beijing & Tianjin & Hebei \\
\hline 2007 & 17.26 & 11.48 & 71.26 \\
2008 & 17.86 & 11.86 & 70.29 \\
2009 & 18.39 & 12.15 & 69.46 \\
2010 & 18.77 & 12.43 & 68.80 \\
2011 & 19.02 & 12.76 & 68.22 \\
2012 & 19.21 & 13.12 & 67.67 \\
2013 & 19.37 & 13.48 & 67.15 \\
2014 & 19.47 & 13.72 & 66.81
\end{tabular}

Table 1 shows the share of the population of the Beijing-Tianjin-Hebei region from 2007 to 2014. It is not difficult to find that, the proportion of the population in Beijing and Tianjin has increased year by year, under the circumstance of the increasing total number of the population in this area; the population of Hebei is declining recent year. This shows that the resident population from the country mostly flows into Beijing and Tianjin, which is consistent with the increasingly serious trend of "the metropolitan malaise" in Beijing and Tianjin.

\section{"population density differentiation"}

It is difficult to discriminate whether the growth of the population is inclusive or extrapolated only by the size of the resident population in the Beijing-Tianjin-Hebei region. Therefore, we use the "population density (person / square kilometer)" to analysis the characteristic of the population. Figure 3 shows the trend of the resident population in the Beijing-Tianjin-Hebei region from 2007 to 2014. It is obviously to find out the resident population in Beijing and Tianjin is far higher than that in Hebei. In 2014, foe example, the resident population density of Beijing and Tianjin is 1311.07 person/square kilometers and 1272.81 person/square kilometers respectively, while the resident population density in Hebei Province is only 395.57 person / square kilometers_—a stark contrast between Hebei and other two cities.

The population density, accordingly, is the index that can explain the "urban congestion". The disparity of "population density" has proved the fact that the development of medium cities is insufficient and the large number of small cities is inefficient. 


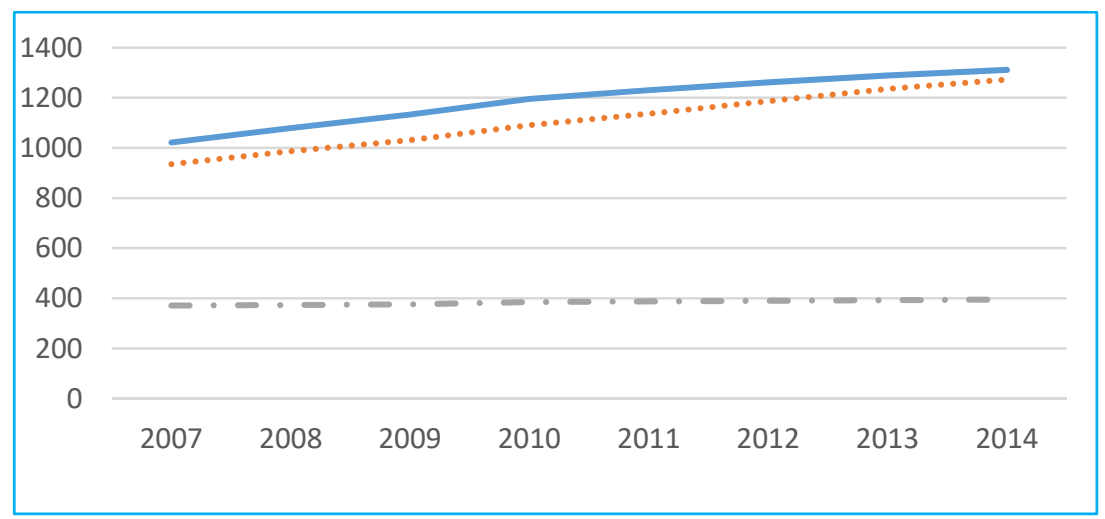

Figure 2: the permanent population density in Beijing-Tianjin-Hebei region (person / square kilometer)

\section{The urban scale system of Beijing, Tianjin and Hebei is unevenly distributed}

Compared with the Yangtze River and the Delta the Pearl River Delta,the status of large cities is over clearly prominent in the Beijing-Tianjin-Hebei region, the number of small cities is much higher than the national level and the number of medium cities is vacant, the feature of the city-size distribution exhibits the sign of "dumbbell". The uneven distribution of the population distribution in the Beijing-Tianjin-Hebei region is not the problem that the size of a single city, its theoretical core is the problem of urban scale distribution.

The Zipf law is the main method of the domestic and foreign research scholars to study the rational distribution of urban scale. The zipf law(Zipf, 1949) argues that the development of city is a kind of a natural stochastic growth, and the distribution of city scale is similar to the power-law distribution, the characteristic of the scale distribution of the urban agglomeration is roughly like a "pyramid".

The Pareto principle of the distribution of city scale can be expressed as $P_{r}=P_{1} / R^{q}, P_{\mathrm{r}}$ indicates the population of the city whose the ranking is $\mathrm{r}$ in the urban agglomeration, $P_{1}$ indicates the population of the most biggest city in the urban agglomeration. $\mathrm{R}$ indicates the ranking of the city $\mathrm{r}, \mathrm{q}$ indicates the Pareto index.

In the case of $\mathrm{q}$ is greater than 1, it indicates the city distribution, in the urban agglomeration, is over centralized - the development of the large city is over outstanding, the development of the small and medium-sized city is insufficient; In the case of $\mathrm{q}$ is less than 1, it indicates that the development of the large city is insufficient and the small and medium-sized cities is over outstanding. When $q$ is equal to 1 , it indicates that the distribution of city scale is consistent with the Zipf's law.

We can get the formula 1 of rank - size rule after the logarithm based on the Pareto principle mentioned, it is indicates that the logarithm of the city rank is linearly decreasing with the logarithm of the population size of the city.

\section{Instructions of data selection}

This paper will use the "resident population" as the caliber to measure the size of the city population of the municipal districts as well as subordinate county-level units of Beijing and Tianjin. Due to lack of the access to the resident population of the corresponding data of Hebei province, the "non-agricultural population" is selected to measure the municipal districts as well as subordinate county-level units of Hebei. Although this will lead to the underestimation of the size of the Hebei, because Hebei is the province which the large number of people move out, the "non-agricultural population", currently, is a good indicator to measure the population of he municipal districts and subordinate county-level units of Hebei.

In this paper, the population data includes the 11 prefecture-level cities and municipalities, 20 county-level cities, 117 counties in the Beijing-Tianjin-Hebei region from 2006 to 2014. 


\section{Analysis of the Zipf regression results}

Table 2 shows the Pareto index of the distribution of the city in the Beijing-Tianjin-Hebei region from 2006 to 2014.

Rank size distribution in Beijing-Tianjin-Hebei region from 2006 to 2014.

It is not difficult to find from Table 2: From the trend of change, q has a tendency of U-shaped;

\begin{tabular}{|c|c|c|c|c|c|c|c|c|}
\hline Year & 2006 & 2008 & 2009 & 2010 & 2011 & 2012 & 2013 & 2014 \\
\hline $\mathrm{q}$ & 1.319509 & 1.243477 & 1.230591 & 1.254777 & 1.241197 & 1.256301 & 1.245092 & 1.28088 \\
\hline
\end{tabular}

taken as a whole, the Q of the Beijing-Tianjin-Hebei region is greater than 1 from 2006 to 2014, indicating that the region is over centralized_- the population of large cities are over prominent and the development of small and medium-sized cities is deficient. The problem, the uneven urban scale system, ought to be solved by stressing the development of the small and medium-sized cities.

The regression result, in accordance with the previous research findings, shows that the power exponent is too high compared with other areas and the distribution of the city scale is unbalanced.

\section{Industry agglomeration and growth of urban population scale}

\section{Industry agglomeration is the main reason for the growth of urban population scale}

The expansion of the urban population, substantially, is the gathering of the labor force in the city. Further, the agglomeration of labor, capital and other productive factors in space is the manifestation of industrial agglomeration.

The degree of the industrial agglomeration directly affects the size of the city population. The gathering of enterprises in the space will increase the demand for labor diversification,accordingly, the matching efficiency of the labor market will be higher, the jobless rate of the local urban will decrease, more external population will be attracted to move into this city so as to the further expansion of the city population. Furthermore, due to a variety of the external economies — - the spillover of knowledge and technology, the sharing of intermediate inputs and labor market, the reduction of transaction costs and the improvement of public infrastructure - the productivity of the large city will be increase.

First, the higher productivity will increase the marginal output of the city's labor force, thereby increase the demand for labor, and attract more external population move for a job. Second, under the role of the market mechanism, higher labor productivity means that the firm's capital can get a higher return and the workers can get higher wages, this could attract more population who wants a better reward.

Momentously, due to the "cumulative causation mechanism" and other complex factors, knowledge spillovers, lower transaction costs, the extent of free trade, market relevance, new technologies, the population of the large city will continue to expand. For example, the increasing population will have positive external effects on knowledge, technology and information exchange, and further increase the labor productivity of large cities, then attract more enterprises and labor into large cities.

In order to test the mechanism of the growth of the population in the cities, we establish a panel regression model as follows:

$$
\ln P O P=\beta_{0}+\beta_{1} \ln S L Q+\beta_{2} \ln T L Q+\varepsilon
$$

Pop expresses the population size of each city and area,SLQ indicates the level of the second industrial agglomeration, and TLQ indicates the level of the third industrial agglomeration.

A great deal of domestic and foreign scholars, customarily, use location quotient as an index to describe the level of the industrial agglomeration, which can truly reflect the spatial distribution of geographical elements as well as the dominant industries and industries. Therefore, this paper uses the location of the indicators to measure the level of the second and third industrial agglomeration in the Beijing-Tianjin-Hebei region. The quotient quotient is expressed as a formula: 


$$
L Q_{i j}=\frac{G_{i j} / \sum_{i=1}^{n} G_{i j}}{\sum_{j=1}^{n} G_{i j} / \sum_{i} \sum_{j} G_{i j}}
$$

LQ indicates the location quotient of the $i$ industry of the $j$ region,GQ indicates the total value of out-put of the $i$ industry of the $j$ region,indicates the total value of out-put of the $i$ industry in the Beijing-Tianjin-Hebei region, indicates the total value of out-put in the Beijing-Tianjin-Hebei region.

\section{The results of empirical analysis}

In this paper, panel data are used to analyze the model. The fixed effect model is suitable after the Housman test. The panel regression results are shown in Table 3.

\begin{tabular}{c|c|c}
\hline C & LNSLQ & LNTLQ \\
\hline $2.23415^{* * *}$ & $0.694148^{* * *}$ & $0.518106^{* * *}$ \\
$(0.054495)$ & $(0.167235)$ & $(0.13339)$ \\
\hline \multirow{2}{*}{ DW } & \multicolumn{2}{|c|}{2.026629} \\
& \multicolumn{2}{|c}{0.959330} \\
& 0.953425
\end{tabular}

From the results of model (2), it is palpable to find out that the second and third industrial agglomeration have a positive impact on the amount of the population, the influence coefficients are 0.167235 and 0.13339 which are significant at $1 \%$ level. This is also consistent with the mechanism we have described earlier- the fundamental reason for the expansion of urban population is industrial agglomeration. The division of labor and technology promote the emergence and the development of the industrial agglomeration, resulting in a variety of external economies, lower transaction costs, knowledge and technology spillovers, the associated industries effects and the shared labor force, to increase the productivity of the enterprises. This will increase the overall and diversity demand for labor in large cities such as Beijing, Shanghai and Tianjin, with the higher labor remuneration, wages. Thus, more population are attracted to come to large city for a job, expanding the scale of large cities.

Therefore, the author believes that through the way, ease and move the enterprise and industry to drive the population move to the small and medium-sized cities, is an important measure, currently, to regulate the population in the Beijing-Tianjin-Hebei region.

\section{The thinking and countermeasures to regulate and control the population of Beijing-Tianjin-Hebei region}

\section{To identify industrial layout in accordance with top-level design}

In the interest of keeping the regional population distribution reasonable and easing the non-core functions of the capital of Beijing, to make clear the function and orientation of Beijing-Tianjin-Hebei and draft the logical industrial layout under the top-level design of BeijingTianjin-Hebei region should be implemented urgently. First, the new strategic positioning of Beijing is the political center, cultural center, international communication center and scientific and technological innovation center. Second, the new strategic positioning of Tianjin is the national advanced manufacturing research and development base, the north international shipping core area, the financial innovation operation demonstration area, and the reform and opening area. Third, the new strategic positioning of Hebei province is an important base of modern commercial logistics in China, an industrial transformation and upgrading test area, a new urbanization and urban-rural integrated demonstration area, and an ecological environment supporting area of Beijing, Tianjin and Hebei region.

The Xiongan new area, the demonstration zone of innovating, which plays an important role in the exploration of economic development and population distribution in the densely populated areas, 
is the support of making the Beijing-Tianjin-Hebei region the new growth pole of the China's economic development and the important fulcrum of Beijing-Tianjin-Hebei urban agglomeration. This also means that, the significance of the Xiongan new area is not just to serve Beijing-Tianjin-Hebei urban agglomeration, but has a more long-term strategic needs, in terms of industrial transfer and layout. The Xiongan new area will carrying on the high energy consumption industry, regional logistics industry and regional professional industry, together with the public service function such as education, medical, training institutions, and part of administrative and public service institutions and some enterprise headquarters.

To speed up the development of small and medium-sized cities in the region, together with improving the quality of development

The massive small cities, with low economies of scale, developed rudely and extensively lacking indispensable plans in the Beijing-Tianjin-Hebei region. Therefore, the small and medium-sized cities in the region should make full use of their own advantages in resources and form a strong support for the industries of big cities. On November 16, 2015, Hebei Province issued a document which specified the function of 11 prefecture-level cities. It not only put forward two cities-Dingzhou and Xinji-as the node cities of characteristic functions, but also proposed the proposal which make 15 small cities around Beijing as the satellite cities, to undertake part of the labor force and promote the coordinated development in the region, the policy should not be too much inclined to small and medium-sized cities. The government should respect and utilize the rules of the market, otherwise it will cause errors in the allocation of resources between regions, reducing the efficiency of the use of resources.

\section{Coordinated development requires co-ordination of population carrying capacity}

The frequent outbreak of the haze crisis, discharging the contaminants recklessly and other issues to remind us: in the process of adjusting population distribution, considering the coordinated development of people and economic society is fundamental. But, quite apart from that, the resources, environment and other factors also should be put in the priority. In the formulation of policies, the author believes that, we should pay attention to the following questions: First, the sum of consumption of the Beijing-Tianjin-Hebei region should not exceed the overall coverage of the region; Second, the development of large cities should not exceed its bearing ceiling; Third, the development of small and medium-sized cities should pay attention to its urban scale effect, the inferior limit of development.

\section{References}

[1]Krugman, P. Confronting the Mystery of Urban Hierarchy[J]. Journal of the Japanese and International Economies, 1996, (10).

[2] Gabaix, X. Zipf's Law for Cities: An Explanation[J]. Quarterly Journal of Economics, 1999, (114).

[3] Soo, K. T. Zipf's Law for Cities: A Cross-Country Investigation[J]. Regional Science and Urban Economics, 2005, (35).

[4] Anderson, G. and Y. G. The Size Distribution of Chinese Cities[J]. Regional Science and Urban Economics, 2005, (6).

[5] Chung, J. and T. Lam. China's"City System"in Flux:Explaining Post-Mao Administrative Changes [J].The China Quarterly, 2004, (12). 